\title{
New integral results using Pólya-Szegö inequality
}

\author{
Ahmed Anber And Zoubir Dahmani
}

\begin{abstract}
In this paper, we use the Riemann-Liouville fractional integral to present recent integral results using new inequalities of PólyaSzegö type.
\end{abstract}

\section{Introduction}

The integral inequalities involving functions of independent variables play a fundamental role in the theory of differential equations. Significant development in this area has been achieved in the last two decades. For more details, we refer to $[3,11,14,15]$ and the references therein. The study of fractional type inequalities is also of great importance. We refer the reader to $[1,16]$ for further information and applications.

Let us now introduce some results that have inspired our work. We consider the functional (see [2])

$$
T(f, g):=\frac{1}{b-a} \int_{a}^{b} f(x) g(x) d x-\frac{1}{(b-a)^{2}} \int_{a}^{b} f(x) d x \int_{a}^{b} g(x) d x,
$$

where $f$ and $g$ are two integrable functions on $[a, b]$. Grüss [10] proved the inequality

$$
|T(f, g)| \leq \frac{(M-m)(N-n)}{4},
$$

provided that $f$ and $g$ are two integrable functions on $[a, b]$ satisfying

$$
m \leq f(x) \leq M, n \leq g(x) \leq N, \quad m, M, n, N \in \mathbb{R}, x \in[a, b] .
$$

Using the Pólya-Szegö inequality (see [17], pp. 213-214)

$$
\frac{\int_{a}^{b} f^{2}(x) d x \int_{a}^{b} g^{2}(x) d x}{\left(\int_{a}^{b} f(x) d x \int_{a}^{b} g(x) d x\right)^{2}} \leq \frac{1}{4}\left(\sqrt{\frac{M N}{m n}}+\sqrt{\frac{m n}{M N}}\right)^{2},
$$

Received March 25, 2013.

2010 Mathematics Subject Classification. 26A33, 26D10.

Key words and phrases. Grüss inequality, fractional integral, Pólya-Szegö inequality. http://dx.doi.org/10.12097/ACUTM.2013.17.15 
Dragomir and Diamond [8] proved that

$$
|T(f, g)| \leq \frac{(M-m)(N-n)}{4(b-a)^{2} \sqrt{m M n N}} \int_{a}^{b} f(x) d x \int_{a}^{b} g(x) d x,
$$

where $f$ and $g$ are two positive integrable functions on $[a, b]$ such that

$$
0<m \leq f(x) \leq M<\infty, 0<n \leq g(x) \leq N<\infty .
$$

For other Grüss type inequalities, see $[5,4,6,7,12,13,18]$ and the references cited therein.

The main aim of this paper is to establish some new fractional integral inequalities of Pólya-Szegö type. Then, we use these results to generate some other fractional integral inequalities.

\section{Description of the fractional calculus}

We introduce some definitions and properties concerning the RiemannLiouville fractional integral operator.

Definition 1. A real valued function $f$ is said to be in the space $C_{\mu}([0, \infty))$, $\mu \in \mathbb{R}$, if there exists a real number $p>\mu$ such that $f(t)=t^{p} f_{1}(t)$, where $f_{1} \in C([0, \infty))$.

Definition 2. A function $f$ is said to be in the space $C_{\mu}^{n}([0, \infty)), n \in \mathbb{N}$, if $f^{(n)} \in C_{\mu}([0, \infty))$.

Definition 3. The Riemann-Liouville fractional integral operator of order $\alpha \geq 0$, for a function $f \in C_{\mu}([0, \infty)), \mu \geq-1$, is defined as

$$
\begin{aligned}
& J^{\alpha} f(t)=\frac{1}{\Gamma(\alpha)} \int_{0}^{t}(t-\tau)^{\alpha-1} f(\tau) d \tau, \quad \alpha>0, t>0, \\
& J^{0} f(t)=f(t) .
\end{aligned}
$$

For the convenience of establishing the results, we give the following property:

$$
J^{\alpha} J^{\beta} f(t)=J^{\alpha+\beta} f(t) .
$$

For the expression (3), when $f(t)=t^{\beta}$, we get another expression that will be used later:

$$
J^{\alpha} t^{\beta}=\frac{\Gamma(\beta+1)}{\Gamma(\alpha+\beta+1)} t^{\alpha+\beta} .
$$

For more details, see $[9,16]$. 


\section{Main results}

Our first result is the following theorem.

Theorem 1. Let $f$ and $g$ be two positive integrable functions on $[0, \infty)$. Suppose that there exist positive real numbers $m, M, n, N$ such that

$0<m \leq f(\tau) \leq M<\infty, 0<n \leq g(\tau) \leq N<\infty, \quad \tau \in[0, t], t>0$.

Then we have

$$
\begin{array}{r}
\left|\frac{t^{\alpha}}{\Gamma(\alpha+1)} J^{\alpha}(f(t) g(t))-J^{\alpha} f(t) J^{\alpha} g(t)\right| \\
\leq \frac{(M-m)(N-n)}{4 \sqrt{m M n N}} J^{\alpha} f(t) J^{\alpha} g(t),
\end{array}
$$

for any $\alpha>0, t>0$.

Remark 2. If we take $\alpha=1$, then we obtain (2) on $[0, t]$.

To prove Theorem 1, we need the following lemma.

Lemma 3. Suppose that $h$ and $l$ are two positive integrable functions on $[0, \infty)$ such that

$$
0<m_{1} \leq h(\tau) \leq M_{1}<\infty, 0<n_{1} \leq l(\tau) \leq N_{1}<\infty, \quad \tau \in[0, t], t>0 .
$$

Then for any $\alpha>0, t>0$ we have

$$
\frac{\left(J^{\alpha} h^{2}(t)\right)\left(J^{\alpha} l^{2}(t)\right)}{\left(J^{\alpha} h(t) l(t)\right)^{2}} \leq \frac{1}{4}\left(\sqrt{\frac{M_{1} N_{1}}{m_{1} n_{1}}}+\sqrt{\frac{m_{1} n_{1}}{M_{1} N_{1}}}\right)^{2} .
$$

Proof. From the condition

$$
\frac{h(\tau)}{l(\tau)} \leq \frac{M_{1}}{n_{1}}, \quad \tau \in[0, t], t>0
$$

we have

$$
\frac{n_{1}}{M_{1}}\left(h^{2}(\tau)\right) \leq h(\tau) l(\tau), \quad \tau \in[0, t], t>0
$$

Multiplying both sides of $(7)$ by $\frac{(t-\tau)^{\alpha-1}}{\Gamma(\alpha)}, \tau \in(0, t)$, and integrating the resulting inequality with respect to $\tau$ over $(0, t)$, we get

$$
\frac{n_{1}}{M_{1} \Gamma(\alpha)} \int_{0}^{t}(t-\tau)^{\alpha-1}(h(\tau))^{2} d \tau \leq \frac{1}{\Gamma(\alpha)} \int_{0}^{t}(t-\tau)^{\alpha-1} h(\tau) l(\tau) d \tau
$$

Therefore,

Now, using the condition

$$
\frac{n_{1}}{M_{1}} J^{\alpha} h^{2}(t) \leq J^{\alpha}(h(t) l(t))
$$

$$
\frac{m_{1}}{N_{1}} \leq \frac{h(\tau)}{l(\tau)}, \quad \tau \in[0, t], t>0
$$


we can write

$$
\frac{m_{1}}{N_{1}} J^{\alpha} l^{2}(t) \leq J^{\alpha}(h(t) l(t))
$$

Multiplying (8) and (9), we obtain

$$
\left(\frac{n_{1}}{M_{1}} \frac{m_{1}}{N_{1}}\right) J^{\alpha} h^{2}(t) J^{\alpha} l^{2}(t) \leq\left(J^{\alpha}(l(\tau) h(\tau))\right)^{2} .
$$

This implies that

$$
\frac{J^{\alpha} h^{2}(t) J^{\alpha} l^{2}(t)}{\left(J^{\alpha} l(t) h(t)\right)^{2}} \leq \frac{M_{1} N_{1}}{m_{1} n_{1}} \leq \frac{1}{4}\left(\sqrt{\frac{M_{1} N_{1}}{m_{1} n_{1}}}+\sqrt{\frac{m_{1} n_{1}}{M_{1} N_{1}}}\right)^{2} .
$$

Lemma is thus proved.

Remark 4. If we take $\alpha=1$, then we obtain Pólya-Szegö inequality on $[0, t]$.

Proof of Theorem 1. Denoting

$$
Q(\tau, \rho):=(f(\tau)-f(\rho))(g(\tau)-g(\rho)),
$$

we have

$$
\begin{aligned}
\int_{0}^{t} \frac{(t-\tau)^{\alpha-1}}{\Gamma(\alpha)} Q(\tau, \rho) d \tau= & \int_{0}^{t} \frac{(t-\tau)^{\alpha-1}}{\Gamma(\alpha)}(f(\tau) g(\tau)+f(\rho) g(\rho) \\
& -f(\tau) g(\rho)-f(\rho) g(\tau)) d \tau \\
= & J^{\alpha}((f(t)) g(t))+\frac{t^{\alpha}}{\Gamma(\alpha+1)} f(\rho) g(\rho) \\
& -g(\rho) J^{\alpha}(f(t))-f(\rho) J^{\alpha}(g(t))
\end{aligned}
$$

Consequently,

$$
\begin{aligned}
& \int_{0}^{t} \int_{0}^{t} \frac{(t-\tau)^{\alpha-1}(t-\rho)^{\alpha-1}}{\Gamma^{2}(\alpha)} Q(\tau, \rho) d \tau d \rho \\
& \quad=2 \frac{t^{\alpha}}{\Gamma(\alpha+1)} J^{\alpha}((f(t)) g(t))-2 J^{\alpha} f(t) J^{\alpha} g(t) .
\end{aligned}
$$

On the other hand, using the Cauchy-Schwartz inequality for double integrals, we can write

$$
\begin{aligned}
& \left|\int_{0}^{t} \int_{0}^{t} \frac{(t-\tau)^{\alpha-1}(t-\rho)^{\alpha-1}}{\Gamma^{2}(\alpha)} Q(\tau, \rho) d \tau d \rho\right| \\
& \leq\left[\int_{0}^{t} \int_{0}^{t} \frac{(t-\tau)^{\alpha-1}(t-\rho)^{\alpha-1}}{\Gamma^{2}(\alpha)} f^{2}(\tau) d \tau d \rho\right. \\
& \quad+\int_{0}^{t} \int_{0}^{t} \frac{(t-\tau)^{\alpha-1}(t-\rho)^{\alpha-1}}{\Gamma^{2}(\alpha)} f^{2}(\rho) d \tau d \rho
\end{aligned}
$$




$$
\begin{aligned}
& \left.\quad-2 \int_{0}^{t} \int_{0}^{t} \frac{(t-\tau)^{\alpha-1}(t-\rho)^{\alpha-1}}{\Gamma^{2}(\alpha)} f(\rho) f(\tau) d \tau d \rho\right]^{\frac{1}{2}} \\
& \times\left[\int_{0}^{t} \int_{0}^{t} \frac{(t-\tau)^{\alpha-1}(t-\rho)^{\alpha-1}}{\Gamma^{2}(\alpha)} g^{2}(\tau) d \tau d \rho\right. \\
& +\int_{0}^{t} \int_{0}^{t} \frac{(t-\tau)^{\alpha-1}(t-\rho)^{\alpha-1}}{\Gamma^{2}(\alpha)} g^{2}(\rho) d \tau d \rho \\
& \left.-2 \int_{0}^{t} \int_{0}^{t} \frac{(t-\tau)^{\alpha-1}(t-\rho)^{\alpha-1}}{\Gamma^{2}(\alpha)} g(\rho) g(\tau) d \tau d \rho\right]^{\frac{1}{2}} .
\end{aligned}
$$

Therefore,

$$
\begin{aligned}
\mid \int_{0}^{t} \int_{0}^{t} & \frac{(t-\tau)^{\alpha-1}(t-\rho)^{\alpha-1}}{\Gamma^{2}(\alpha)} Q(\tau, \rho) d \tau d \rho \mid \\
\leq & 2\left[\frac{t^{\alpha}}{\Gamma(\alpha+1)} J^{\alpha} f^{2}(t)-\left(J^{\alpha} f(x)\right)^{2}\right]^{\frac{1}{2}} \\
& \times\left[\frac{t^{\alpha}}{\Gamma(\alpha+1)} J^{\alpha} g^{2}(t)-\left(J^{\alpha} g(x)\right)^{2}\right]^{\frac{1}{2}}
\end{aligned}
$$

Using Lemma 3, we get

$$
\begin{aligned}
\frac{t^{\alpha}}{\Gamma(\alpha+1)} J^{\alpha} f^{2}(x) & \leq \frac{1}{4}\left(\sqrt{\frac{M}{m}}+\sqrt{\frac{m}{M}}\right)^{2}\left(J^{\alpha} f(x)\right)^{2} \\
& =\frac{(M+m)^{2}}{4 m M}\left(J^{\alpha} f(x)\right)^{2} .
\end{aligned}
$$

Hence

$$
\frac{t^{\alpha}}{\Gamma(\alpha+1)} J^{\alpha} f^{2}(x)-\left(J^{\alpha} f(x)\right)^{2} \leq\left(\frac{(M+m)^{2}}{4 m M}-1\right)\left(J^{\alpha} f(x)\right)^{2},
$$

that is

$$
\frac{t^{\alpha}}{\Gamma(\alpha+1)} J^{\alpha} f^{2}(x)-\left(J^{\alpha} f(x)\right)^{2} \leq \frac{(M-m)^{2}}{4 m M}\left(J^{\alpha} f(x)\right)^{2} .
$$

In a similar fashion, we obtain

$$
\frac{t^{\alpha}}{\Gamma(\alpha+1)} J^{\alpha} g^{2}(x)-\left(J^{\alpha} g(x)\right)^{2} \leq \frac{(N-n)^{2}}{4 n N}\left(J^{\alpha} g(x)\right)^{2} .
$$

Combining (10), (11), (12) and (13), we deduce the desired inequality (5). 
Our second result is the following.

Theorem 5. Let $f$ and $g$ be two positive integrable functions on $[0, \infty)$. Suppose that there exist positive real numbers $m, n, M, N$ such that (4) holds. Then we have

$$
\begin{gathered}
\mid \frac{t^{\alpha}}{\Gamma(\alpha+1)} J^{\beta}(f(t) g(t))+\frac{t^{\beta}}{\Gamma(\beta+1)} J^{\alpha}(f(t) g(t)) \\
-J^{\alpha} f(t) J^{\beta} g(t)-J^{\beta} f(t) J^{\alpha} g(t) \mid \\
\leq \frac{(M-m)(N-n)}{2 \sqrt{m M n N}} J^{\alpha} f(t) J^{\beta} g(t)
\end{gathered}
$$

for any $\alpha>0, \beta>0, t>0$.

To prove this theorem, we need the following lemma which is another generalization of Pólya-Szegö inequality (1).

Lemma 6. Suppose that $h$ and $l$ are two positive integrable functions on $[0, \infty)$ such that $(6)$ holds. Then for any $\alpha>0, \beta>0, t>0$, we have

$$
\frac{\left(J^{\alpha} h^{2}(t)\right)\left(J^{\beta} l^{2}(t)\right)}{\left(J^{\alpha} h(t) l(t)\right)\left(J^{\beta} h(t) l(t)\right)} \leq \frac{1}{4}\left(\sqrt{\frac{M_{1} N_{1}}{m_{1} n_{1}}}+\sqrt{\frac{m_{1} n_{1}}{M_{1} N_{1}}}\right)^{2} .
$$

Proof. By the condition

$$
\frac{m_{1}}{N_{1}} \leq \frac{h(\tau)}{l(\tau)}, \quad \tau \in[0, t], t>0,
$$

we get

$$
\frac{m_{1}}{N_{1}} J^{\beta} l^{2}(t) \leq J^{\beta}(h(t) l(t)) .
$$

Thanks to (8) and (16) we obtain (15).

Proof of Theorem 5. We have

$$
\begin{aligned}
\int_{o}^{t} \int_{0}^{t} \frac{(t-\tau)^{\alpha-1}(t-\rho)^{\beta-1}}{\Gamma(\alpha) \Gamma(\beta)} Q(\tau, \rho) d \tau d \rho \\
=\frac{t^{\alpha}}{\Gamma(\alpha+1)} J^{\beta}((f(t)) g(t))+\frac{t^{\beta}}{\Gamma(\beta+1)} J^{\alpha}((f(t)) g(t)) \\
\quad-J^{\alpha} f(t) J^{\beta} g(t)-J^{\beta} f(t) J^{\alpha} g(t) .
\end{aligned}
$$

As in the proof of Theorem 1, using Cauchy-Schwartz inequality for double integrals, we find that

$$
\left|\int_{0}^{t} \int_{0}^{t} \frac{(t-\tau)^{\alpha-1}(t-\rho)^{\beta-1}}{\Gamma(\alpha) \Gamma(\beta)} Q(\tau, \rho) d \tau d \rho\right|
$$




$$
\begin{aligned}
\leq & {\left[\int_{0}^{t} \int_{0}^{t} \frac{(t-\tau)^{\alpha-1}(t-\rho)^{\beta-1}}{\Gamma(\alpha) \Gamma(\beta)} f^{2}(\tau) d \tau d \rho\right.} \\
& +\int_{0}^{t} \int_{0}^{t} \frac{(t-\tau)^{\alpha-1}(t-\rho)^{\beta-1}}{\Gamma(\alpha) \Gamma(\beta)} f^{2}(\rho) d \tau d \rho \\
& \left.-2 \int_{0}^{t} \int_{0}^{t} \frac{(t-\tau)^{\alpha-1}(t-\rho)^{\beta-1}}{\Gamma(\alpha) \Gamma(\beta)} f(\rho) f(\tau) d \tau d \rho\right]^{\frac{1}{2}} \\
\times & {\left[\int_{0}^{t} \int_{0}^{t} \frac{(t-\tau)^{\alpha-1}(t-\rho)^{\beta-1}}{\Gamma(\alpha) \Gamma(\beta)} g^{2}(\tau) d \tau d \rho\right.} \\
& +\int_{0}^{t} \int_{0}^{t} \frac{(t-\tau)^{\alpha-1}(t-\rho)^{\beta-1}}{\Gamma(\alpha) \Gamma(\beta)} g^{2}(\rho) d \tau d \rho \\
& \left.-2 \int_{0}^{t} \int_{0}^{t} \frac{(t-\tau)^{\alpha-1}(t-\rho)^{\beta-1}}{\Gamma(\alpha) \Gamma(\beta)} g(\rho) g(\tau) d \tau d \rho\right]^{\frac{1}{2}}
\end{aligned}
$$

Thus

$$
\begin{aligned}
\mid \int_{0}^{t} \int_{0}^{t} & \frac{(t-\tau)^{\alpha-1}}{\Gamma(\alpha)} \frac{(t-\rho)^{\alpha-1}}{\Gamma(\alpha)} Q(\tau, \rho) d \tau d \rho \mid \\
\leq & {\left[\frac{t^{\alpha}}{\Gamma(\alpha+1)} J^{\beta} f^{2}(t)+\frac{t^{\beta}}{\Gamma(\beta+1)} J^{\alpha} f^{2}(t)-2 J^{\alpha} f(t) J^{\beta} f(t)\right]^{\frac{1}{2}} } \\
& \times\left[\frac{t^{\alpha}}{\Gamma(\alpha+1)} J^{\beta} g^{2}(t)+\frac{t^{\beta}}{\Gamma(\beta+1)} J^{\alpha} g^{2}(t)-2 J^{\alpha} g(t) J^{\beta} g(t)\right]^{\frac{1}{2}} .
\end{aligned}
$$

Using Lemma 6, we may state that

$$
\frac{t^{\alpha}}{\Gamma(\alpha+1)} J^{\beta} f^{2}(t)-J^{\alpha} f(t) J^{\beta} f(t) \leq \frac{(M-m)^{2}}{4 m M} J^{\alpha} f(t) J^{\beta} f(t)
$$

and

$$
\frac{t^{\beta}}{\Gamma(\beta+1)} J^{\alpha} f^{2}(t)-J^{\alpha} f(t) J^{\beta} f(t) \leq \frac{(M-m)^{2}}{4 m M} J^{\alpha} f(t) J^{\beta} f(t) .
$$

For the function $g$, we have

$$
\frac{t^{\alpha}}{\Gamma(\alpha+1)} J^{\beta} g^{2}(t)-J^{\alpha} g(t) J^{\beta} g(t) \leq \frac{(N-n)^{2}}{4 n N} J^{\alpha} g(t) J^{\beta} g(t)
$$

and

$$
\frac{t^{\beta}}{\Gamma(\beta+1)} J^{\alpha} g^{2}(t)-J^{\alpha} g(t) J^{\beta} g(t) \leq \frac{(N-n)^{2}}{4 n N} J^{\alpha} g(t) J^{\beta} g(t) .
$$

Now, in view of (17) and (18)-(22), we obtain (14). 
Remark 7. Applying Theorem 5, for $\alpha=\beta$ we obtain Theorem 1 , and for $\alpha=\beta=1$ we obtain (4) on $[0, t]$.

\section{References}

[1] G. A. Anastassiou, Fractional Differentiation Inequalities, Springer, Dordrecht, 2009.

[2] P.L. Chebyshev, Sur les expressions approximatives des intégrales définies par les autres prises entre les mêmes limites, Proc. Math. Soc. Charkov 2 (1882), 93-98.

[3] P. Cerone and S. S. Dragomir, A refinement of the Grüss inequality and applications, Tamkang J. Math. 38 (2007), 37-49.

[4] Z. Dahmani and L. Tabharit, On weighted Grüss type inequalities via fractional integration, J. Adv. Res. Pure Math. 2 (2010), 31-38.

[5] Z. Dahmani, L. Tabharit, and S. Taf, New generalisation of Grüss inequality using Riemann-Liouville fractional integrals, Bull. Math. Anal. Appl. 2 (2010), 93-99.

[6] S.S. Dragomir, A generalization of Grüss's inequality in inner product spaces and applications, J. Math. Anal. Appl. 237 (1999), 74-82.

[7] S. S. Dragomir, Some integral inequalities of Grüss type, Indian J. Pure Appl. Math. 31 (2000), 397-415.

[8] S. S. Dragomir and N. T. Diamond, Integral inequalities of Grüss type via Pólya-Szegö and Shisha-Mond results, East Asian Math. J. 19 (2003), 27-39.

[9] R. Gorenflo and F. Mainardi, Fractional Calculus: Integral and Differential Equations of Fractional Order, CISM Courses and Lectures 378, Springer, Vienna, 1997.

[10] G. Grüss, Über das Maximum des absoluten Betrages von $\frac{1}{b-a} \int_{a}^{b} f(x) g(x) d x-$ $\frac{1}{(b-a)^{2}} \int_{a}^{b} f(x) d x \int_{a}^{b} g(x) d x$, Math. Z. 39 (1935), 215-226.

[11] S. Mazouzi and F. Qi, On an open problem regarding an integral inequality, J. Inequal. Pure Appl. Math. 4 (2003), No. 2, Article 31.

[12] A. McD. Mercer, An improvement of the Grüss inequality, J. Inequal. Pure Appl. Math. 6 (2005), No. 4, Article 93.

[13] A. McD. Mercer and P. Mercer, New proofs of the Grüss inequality, Aust. J. Math. Anal. Appl. 1 (2004), No.2, Art. 12.

[14] D. S. Mitrinović, J. E. Pečarić, and A. M. Fink, Classical and New Inequalities in Analysis, Mathematics and its Applications (East European Series) 61, Kluwer Academic Publishers Group, Dordrecht, 1993.

[15] B. G. Pachpatte, On multidimensional Grüss type integral inequalities, J. Inequal. Pure Appl. Math. 3 (2002), No. 2, Article 27.

[16] I. Podlubny, Fractional Differential Equations, Mathematics in Science and Engineering 198, Academic Press, San Diego, 1999.

[17] G. Pólya and G. Szegö, Aufgaben und Lehrsätze aus der Analysis. Band 1, Die Grundlehren der mathmatischen Wissenschaften 19, J. Springer, Berlin, 1925.

[18] M.Z. Sarikaya, N. Aktan, and H. Yildirim, On weighted Čebyšev-Grüss type inequalities on time scales, J. Math. Inequal. 2 (2008), 185-195.

Department of Mathematics, University of UStO, Oran, Algeria

Laboratory of Pure and Applied Mathematics, Faculty SEI, UMAB, University of Mostaganem, Algeria

E-mail address: zzdahmani@yahoo.fr 